\title{
Statistical Mechanics of a One-dimensional Lattice Gas with Exponential-polynomial Interactions
}

\author{
K. S. Viswanathan* \\ Institut des Hautes Etudes Scientifiques, F-91440 Bures-sur-Yvette, France
}

\begin{abstract}
Some properties of the transfer-matrix for a one-dimensional classical lattice-gas with exponential-polynomial pair interactions are studied using Hilbert space techniques.
\end{abstract}

\section{Introduction and Statement of Results}

We are concerned here with the statistical mechanics of a classical, one-dimensional lattice-gas, or equivalently of a spin system with exponentially decreasing pair interactions of the type

$$
\varphi_{1}(n)=\lambda^{n} \sum_{i=0}^{p} c_{i} n^{i} \quad(0<\lambda<1)
$$

as well as potentials which are a finite sum of decreasing exponentials,

$$
\varphi_{2}(n)=\sum_{i=1}^{k} c_{i} \lambda_{i}^{n} \quad(0<\lambda<1)
$$

potential (1.1) will be termed exponential-polynomial type. Ruelle [1] ${ }^{1}$ has established the absence of phase transitions in one-dimensional systems with translationally invariant two-body interactions that satisfy the condition

$$
\sum_{i \in \mathbb{N}} i|\varphi(0, i)|<\infty
$$

where $\mathbb{N}$ is the set of all integers $>0$.

* Permanent address: Department of Physics, Simon Fraser University, Burnaby 2, British Columbia, Canada.

1 Ruelle's results actually extend to many-body translationally invariant interactions which satisfy the following criterion

$$
\sum_{l>0} \sum_{0<i_{1}<i_{2}<\ldots<i_{l}} i_{l}\left|\varphi^{(l+1)}\left(0, i_{1}, i_{2}, \ldots, i_{l}\right)\right|<\infty
$$

where $\varphi^{(l+1)}$ is the $(l+1)$ body potential. 
Furthermore Ruelle [1] has shown that the study of the statistical mechanics of one-dimensional lattice systems that satisfy (1.3) is greatly simplified by introducing the following operator $\mathscr{L}$ on the space $C\left(K_{+}\right)$of functions continuous on $K_{+}=[0,1]^{\mathbb{N}}$. If $f \in C\left(K_{+}\right), x \in K_{+}$; i.e., $x=\left\{x_{i}\right\}_{i \in \mathbb{N}}$ :

$$
\mathscr{L} f(x)=f(0, x)+\gamma \exp \left(-\sum_{i \in \mathbb{N}} x_{i} \varphi(i)\right) f(1, x) .
$$

The operator $\mathscr{L}$ defined above is continuous but not compact. In order to introduce a compact operator one proceeds as follows [2]. One first notes that in [1], one utilizes the Banach space character of $C\left(K_{+}\right)$which contains the functions $\mathscr{L}^{n} \mathbb{1}, n \in \mathbb{N}$. However, $\mathscr{L}^{n} \mathbb{1}$ is an entire function of $\sum_{i \in \mathbb{N}} x_{i} \varphi(i)$. This suggests that we consider changing to a variable $z(x)$ defined by

$$
z(x)=\sum_{k=1}^{\infty} x_{k} \lambda^{k} ; \quad x=\left\{x_{k}\right\}_{k \in \mathbb{N}} \in K_{+} .
$$

Let $D$ be a closed disk with center at the origin and of radius $R>\lambda /(1-\lambda)$. Let, further,

$$
A_{\lambda}(D)=\left(f: f \in C\left(K_{+}\right), f(z)=\varphi(z(x))\right.
$$

where $\varphi(z)$ is an analytic function in a circle of radius $|z|<R$ such that, if $\varphi(z)=$ $\sum_{n=0}^{\infty} C_{n} z^{n}$, then $\sum_{n=1}^{\infty} R^{2 n}\left|C_{n}\right|^{2}<\infty$. Then the restriction $\mathscr{L}_{D}$ of the operator $\mathscr{L}$ acting on $A_{\lambda}(D)$ can be seen to be defined by

$$
\mathscr{L}_{D} \varphi(z)=\varphi(\lambda z)+\gamma \exp (-c z) \varphi(\lambda+\lambda z) .
$$

Proposition 1. $\mathscr{L}_{D} A_{\lambda}(D) \subset A_{\lambda}(D)$.

The proof follows immediately from the above definition.

Definition. Define on $A_{\lambda}(D)$ a scalar product

$$
\langle f \mid g\rangle=\sum_{n=0}^{\infty} R^{2 n} \bar{C}_{n} \gamma_{n}
$$

where $f(z)=\sum C_{n} Z^{n}$ and $g(z)=\sum \gamma_{n} z^{n}$. Then $A_{\lambda}(D)$ becomes a Hilbert space $\mathscr{H}(D)$. Ferrero [2] has shown that, provided $0<\lambda<1 / 2, \mathscr{L}$ in (1.6) is compact. It is not necessary in what follows to restrict $\lambda<1 / 2$. We shall require only that $0<\lambda<1$. In this article we establish some further properties of $\mathscr{L}$ and elucidate its connection with the transfer matrix. In particular we establish the following theorems.

Theorem 1. The operator $\mathscr{L}_{D}^{N}$ in (1.6) is a trace-class operator $\forall N \geqq 1$, and its largest eigenvalue coincides with the largest eigenvalue of $\mathscr{L}^{N}$ on $C\left(K_{+}\right)$(which is unique and positive).

Corollary 1. The principal eigenvector of $\mathscr{L}$ is of the form $h(x)=\varphi(z(x))$, where $\varphi(z)$ is an entire function of $z$.

Corollary 2. The largest eigenvalue of $\mathscr{L}$ on $C\left(K_{+}\right)$depends analytically on $\gamma$ in the neighborhood of $\gamma$ real. 
In what follows we drop the suffix $D$ on the operator $\mathscr{L}_{D}$.

Theorem 2. $\operatorname{Tr}\left(\mathscr{L}^{N}\right)$ is, up to a multiplicative constant $\left(1-\lambda^{N}\right)$, the partition function $Q_{N}$ for a one-dimensional lattice-gas containing $N$-sites interacting through a pair potential

$$
\varphi(n)=c \lambda^{n}
$$

with periodic boundary conditions.

By this we mean: A given site $i(0 \leqq i \leqq N)$ interacts with all the sites of $\mathbb{Z}$ to its right. $\left(\mathbb{Z}=\right.$ the set of integers $\geqq 0$.) The occupation $x_{i}$ for $i \geqq N$ is determined by

$$
x_{i+N}=x_{i}
$$

where

$$
x_{i}=\left\{\begin{array}{llll}
0 & \text { if site } & i & \text { is empty } \\
1 & \text { if site } & i & \text { is occupied }
\end{array}\right.
$$

Theorem 3. We form the function

$$
\Xi(z)=\exp \left(\sum_{N=1}^{\infty}\left(z^{N} / N\right) Q_{N}\right)
$$

where

$$
Q_{N}=\left(1-\lambda^{N}\right) \operatorname{Tr} \mathscr{L}^{N} .
$$

Then $\Xi(z)$, which is analytic in the neighborhood of $z=0$, extends by analytic continuation to a meromorphic function in the entire z-plane.

In Section III, we extend our results to systems with exponential-polynomial interactions of the form (1.1). The operator $\mathscr{L}$ now acts on a Hilbert-space $\mathscr{H}(D)$ of functions of $(p+1)$ complex variables, holomorphic on open polydisc $D_{(p+1)}$.

$$
\mathscr{L} f(z)=f(\lambda A z)+\gamma \exp (-\tilde{c} \cdot z) f(\lambda(A z+I))
$$

where

$$
\begin{aligned}
& z=\left[\begin{array}{c}
z_{0} \\
z_{1} \\
z_{2} \\
\vdots \\
z_{p}
\end{array}\right] \\
& \left.\begin{array}{rl}
(A)_{i j} & =\left(\begin{array}{l}
i \\
j
\end{array}\right) \text { if } j \leqq i \\
& =0 \text { otherwise }
\end{array}\right\} 0 \leqq i, j \leqq p,
\end{aligned}
$$


i.e. $A$ is a $(p+1) \times(p+1)$ triangular matrix.

$$
I=\left[\begin{array}{c}
1 \\
1 \\
\vdots \\
1
\end{array}\right] \text { and } \tilde{c} \circ z=\sum_{i=0}^{p} c_{i} z_{i}
$$

Finally we indicate how some obvious generalizations can be made to systems with pair-interactions of the form 1.2.

\section{Proof of Theorems 1-3}

Lemma 1. The operator $\mathscr{L}$ defined in (1.6) admits the following representation.

$$
\mathscr{L}=\sum_{x=0,1} \sum_{n=0}^{\infty} \lambda^{n}\left|\varphi_{n}^{(x)}\right\rangle\left\langle\psi_{n}^{(x)} \mid \cdot\right\rangle_{\mathscr{H} *}
$$

where

$$
\left|\varphi_{n}^{(x)}\right\rangle=\gamma^{x} e^{-x c z} z^{n}
$$

and

$$
\begin{aligned}
\left\langle\psi_{n}^{(x)} \mid f\right\rangle_{\mathscr{H}^{*}} & =1 / 2 \pi i \oint_{\partial_{0} D} f(z)(z-\lambda x)^{-(n-1)} d z \\
& =1 / n ! f^{n}(\lambda x) \equiv T_{x}^{n} f .
\end{aligned}
$$

Note that $\left\{\varphi_{n}^{(0)}\right\}_{n \geq 0}$ is a complete orthonormal basis in $\mathscr{H}(D)$. Furthermore $\left\langle\psi_{n}^{(x)} \mid f\right\rangle_{\mathscr{H}^{*}}$ is a linear functional in the dual $\mathscr{H}^{*}$ of $\mathscr{H}(D)$ and hence it follows from Riesz' theorem that there exists a unique $\xi_{n} \in \mathscr{H}(D)$ such that

$$
\left\langle\psi_{n}^{(x)} \mid f\right\rangle_{\mathscr{H}^{*}}=\left\langle\xi_{n}^{x} \mid f\right\rangle_{\mathscr{H}(D)} \quad \forall f \in \mathscr{H}(D)
$$

and that

$$
\left\|\xi_{n}^{(x)}\right\|_{\mathscr{H}(D)}=\left\|T_{x}^{n}\right\|_{\mathscr{H}^{*}} .
$$

Proof of Lemma 1. Let $f(z) \in \mathscr{H}(D)$. Then

$$
\begin{aligned}
\mathscr{L} f(z) & =\sum \lambda^{n}\left|\varphi_{n}^{(x)}\right\rangle\left\langle\psi_{n}^{(x)} \mid f\right\rangle_{\mathscr{H} *} \\
& =\sum_{n \geqq 0} \lambda^{n} z^{n} f^{n}(0) / n !+\gamma \sum_{n \geqq 0} e^{-c z}(\lambda z)^{n} f^{n}(\lambda) / n ! \\
& =f(\lambda z)+\gamma e^{-c z} f(\lambda+\lambda z) .
\end{aligned}
$$

Proof of Theorem 1. Recall $[3,4]$ that an operator $A$ is of trace class if and only if

$$
\operatorname{Tr}[A]<\infty
$$

where $[A]=+\sqrt{A^{*} A}$. 
Now by Riesz' theorem, $\mathscr{L}$ can be written as

$$
\mathscr{L}=\sum \lambda^{n}\left|\varphi_{n}^{(x)}\right\rangle\left\langle\xi_{n}^{(x)} \mid \cdot\right\rangle_{\mathscr{H}(D)} .
$$

Denote $\operatorname{Tr}[\mathscr{L}]$ by $\tau(\mathscr{L})$, then

$$
\begin{aligned}
\tau(\mathscr{L}) & =\operatorname{Tr}\left[\sum \lambda^{n}\left|\varphi_{n}^{(x)}\right\rangle\left\langle\xi_{n}^{(x)} \mid \cdot\right\rangle\right] \\
& \leqq \sum_{n, x} \lambda^{n} \tau\left(\left|\varphi_{n}^{(x)}\right\rangle\left\langle\xi_{n}^{(x)} \mid \cdot\right\rangle\right) \\
& =\sum_{n, x} \lambda^{n}\left\|\varphi_{n}^{(x)}\right\|_{\mathscr{H}(D)}\left\|\xi_{n}^{(x)}\right\|_{\mathscr{H}(D)} .
\end{aligned}
$$

The following estimates are easily verified.

$$
\begin{aligned}
\left\|\varphi_{n}^{(0)}\right\| & =R^{n} \\
\left\|\varphi_{n}^{(1)}\right\| & \leqq \gamma R^{n} \exp \left(c^{2} R^{2} / 2\right) \\
\left\|\psi_{n}^{(0)}\right\| & \leqq 1 / R^{n} \\
\left\|\psi_{n}^{(1)}\right\| & \leqq R^{-n}(1-\lambda / R)^{-(n+1)}
\end{aligned}
$$

substituting these estimates in (2.8) one finds that

$$
\tau(\mathscr{L}) \leqq 1 /(1-\lambda)\left\{1+R \gamma \exp \left(C^{2} R^{2} / 2\right) /(R-\lambda /(1-\lambda))\right\}
$$

provided $R>\lambda /(1-\lambda)$. This is precisely the restriction we had imposed on the radius $R$ of the disk at the beginning. Compactness of $\mathscr{L}$ follows at once as a corollary of Theorem 1 .

\section{Proposition 2.}

$\operatorname{Tr} \mathscr{L}=1 /(1-\lambda)\left\{1+\gamma \exp \left(-c \sum_{n=1}^{\infty} \lambda^{n}\right)\right\}$.

Remark. $(1-\lambda) \operatorname{Tr} \mathscr{L}$ can be interpreted as the partition function for a system with one site (site 1 ) interacting with all other sites $n \geqq 1$ to the right with the pair potential (1.7) and $x_{1+n}=x_{n}$. For all $n \geqq 1$.

Proof. Choose an orthonormal basis $\left\{x_{n}\right\}$ in $\mathscr{H}(D)$. Then

$$
\begin{aligned}
\operatorname{Tr} \mathscr{L} & =\sum\left\langle x_{n}|\mathscr{L}| x_{n}\right\rangle \\
& =\sum_{m, x} \lambda^{m}\left\langle\psi_{m}^{(x)} \mid \varphi_{n}^{(x)}\right\rangle_{\mathscr{H} *} \\
& =\sum_{m, x} \gamma^{x} \lambda^{m} \oint_{\partial^{0} D} e^{-x c z} \cdot z^{m}(z-\lambda x)^{-(m+1)} d z / 2 \pi i \\
& =\sum_{x} \gamma^{x} /(1-\lambda) \oint_{\partial_{0} D} e^{-x c z}(z-\lambda x /(1-\lambda))^{-1} d z / 2 \pi i \\
& =1 /(1-\lambda)\left\{1+\gamma \exp -\left(c \sum_{n=1}^{\infty} \lambda^{n}\right)\right\} .
\end{aligned}
$$

(2.11d) follows from the fact that $\sum(\lambda z /(z-\lambda))^{m}$ is uniformly convergent for $|z|>\lambda /(1-\lambda)(0<\lambda<1)$. 
Proof of Theorem 2.

$$
\begin{aligned}
& \operatorname{Tr} \mathscr{L}^{N}=\sum_{\left\{x_{1}\right\}} \operatorname{Tr}\left(\mathscr{L}_{x_{1}} \mathscr{L}_{x_{2}} \ldots \mathscr{L}_{x_{N}}\right) \\
& =\sum_{\left\{x_{t}\right\}} \sum_{\left\{n_{t}\right\}} \prod_{i}\left(\lambda^{n_{2}}\right)\left\langle\psi_{n_{N}}^{\left(x_{N}\right)} \mid \varphi_{n_{1}}^{\left(x_{1}\right)}\right\rangle_{\mathscr{H} *}\left\langle\psi_{n_{1}}^{\left(x_{1}\right)} \mid \varphi_{n_{2}}^{\left(x_{2}\right)}\right\rangle_{\mathscr{H}^{*}} \\
& \times \ldots\left\langle\psi_{n_{N-1}}^{\left(x_{N}-1\right)} \mid \varphi_{n_{N}}^{\left(x_{N}\right)}\right\rangle_{\mathscr{H}} * \\
& =\sum_{\left\{x_{i}\right\}}\left(\gamma^{\sum x_{k}}\right) \sum_{\left\{n_{l}\right\}} \prod_{i}\left(\lambda^{n_{i}}\right) \oint_{c_{0} D} \prod_{k=1}^{N}\left(d z_{k}\right) \\
& \cdot(2 \pi i)^{-N} \exp \left(-c \sum_{k=1}^{N} x_{k+1} z_{k}\right) \prod_{k=1}^{N}\left(z_{k}\right)^{n_{k+1}} / \prod_{k=1}^{N}\left(z_{k}-\lambda x_{k}\right)^{n_{k}+1} .
\end{aligned}
$$

In above $\mathscr{L}_{0}$ is that part of $\mathscr{L}$ that corresponds to $x=0$ in $(2.1)$ and $\mathscr{L}_{1}$ is the part with $x=1$.

$$
x_{k+N}=x_{k} \text { and } n_{k+N}=n_{k} .
$$

In $(2.12 c) \partial_{0} D$ is the distinguished boundary of $D^{N}$; i.e.

$$
\partial_{0} D=\partial_{0} D_{1} \times \partial_{0} D_{2} \times \ldots \times \partial_{0} D_{N} .
$$

The $N$-fold summation is uniformly convergent if $\lambda\left|z_{k}\right|<\left|z_{k+1}-\lambda\right|(k=1, \ldots, N)$ and in particular if the radii $R_{k}$ of $\partial_{0} D_{k}$ are all equal and such that $R_{k}=R_{0}>$ $\lambda /(1-\lambda) ;(\forall k)$. Thus

$$
\begin{aligned}
\operatorname{Tr} \mathscr{L}^{N}= & \sum_{\left\{x_{i}\right\}} \gamma^{\sum x_{k}} \oint_{\hat{C}_{0} D} \prod_{k} d z_{k}(2 \pi i)^{N} \\
& \cdot \exp \left(-c \sum_{k} x_{k+1} z_{k}\right) / \prod_{k=1}^{N}\left(z_{k+1}-\lambda x_{k+1}-\lambda z_{k}\right) .
\end{aligned}
$$

Define a new variable

$$
z_{k+1}-z_{k}=w_{k+1} \quad \text { with } \quad w_{k+N}=w_{k} \quad(k=1, \ldots, N) .
$$

In matrix notation $(2.15)$ reads

$$
A Z=W
$$

where

$$
\begin{aligned}
& N \text { Columns }
\end{aligned}
$$

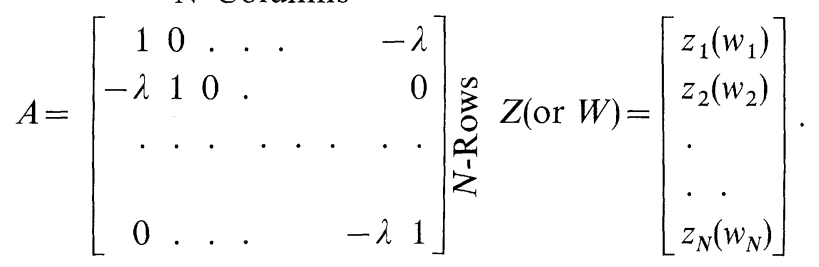

Inverting (2.16) we get

$$
\begin{gathered}
z_{i}=1 /\left(1-\lambda^{N}\right) \sum_{k=1}^{N} w_{k+i} \lambda^{N-k}, \\
\prod_{i}\left(d z_{i}\right)=1 /\left(1-\lambda^{N}\right) \prod_{i} d w_{i} .
\end{gathered}
$$


Inserting (18) and (19) into (2.14), one gets

$$
\begin{aligned}
\operatorname{Tr} \mathscr{L}^{N}= & 1 /\left(1-\lambda^{N}\right) \sum_{\left\{x_{i}\right\}} \oint_{\partial_{0} D^{\prime}} \prod_{k} d w_{k}\left(\gamma^{\sum^{k} x_{k}}\right) \\
& \cdot \exp \left(-c /\left(1-\lambda^{N}\right) \sum_{k} x_{k+1} \sum_{i} w_{k+1} \lambda^{N-i}\right)(2 \pi i)^{-N} / \prod_{k=1}^{N}\left(w_{k}-\lambda x_{k}\right) .
\end{aligned}
$$

Clearly, if $\left|z_{k}\right|>\frac{\lambda}{(1-\lambda)}(\forall k)$, on applying Cauchy's theorem we pick up the contribution from the poles at $w_{k}=\lambda x_{k} \forall k$, and we obtain the following.

$$
\begin{aligned}
\operatorname{Tr} \mathscr{L}^{N} & =1 /\left(1-\lambda^{N}\right) \sum_{\left\{x_{i}\right\}}\left(\prod_{k} \lambda^{x_{k}}\right) \exp \left(-c \lambda /\left(1-\lambda^{N}\right) \sum_{k=1}^{N} x_{k+1} \sum_{i=1}^{N} \lambda^{N-i} x_{k+i}\right) \\
& =1 /\left(1-\lambda^{N}\right) \sum_{\left\{x_{i}\right\}}\left(\prod_{k} \gamma^{x_{k}}\right) \exp \left(-c \sum_{k=1}^{N} x_{k+1} \sum_{l=1}^{N} x_{k+i} \sum_{l=0}^{\infty} \lambda^{(N l+N-i+1)}\right) .
\end{aligned}
$$

Let $k+i(\bmod N)=s,(0 \leqq k, i \leqq N)$ and $N+1-i=t$. Imposing the condition $x_{i+N}=x_{i}(0 \leqq i \leqq N)$ we can write $(2.21 \mathrm{~b})$ as

$$
\operatorname{Tr} \mathscr{L}^{N}=1 /\left(1-\lambda^{N}\right) \sum_{\left\{x_{i}\right\}} \exp \left(-c \sum_{s=1}^{N} x_{s} \sum_{t=1}^{\infty} x_{s+t} \lambda^{t}\right)\left(\gamma^{k} x_{k}\right) .
$$

Thus $\operatorname{Tr} \mathscr{L}^{N}$ is $\left(1-\lambda^{N}\right)$ times the partition function for a lattice gas of $N$ sites subject to the boundary conditions imposed earlier.

Proof of Theorem 3. From (1.10) and (1.11) it follows that

$$
\begin{aligned}
\Xi(z) & =\exp \left(\sum_{N=1}^{\infty}\left(z^{N} / N_{N}\right)\left(1-\lambda^{N}\right) \operatorname{Tr} \mathscr{L}^{N}\right) \\
& =\exp \left(\sum_{N=1}^{\infty}\left(z^{N} / N_{N}\right)\left(1-\lambda^{N}\right) \sum_{\{k\}} \lambda_{k}^{N}\right)
\end{aligned}
$$

where $\left\{\lambda_{k}\right\}$ are the eigenvalues of $\mathscr{L}$ repeated according to their multiplicity. For convenience we assume that the set $\left\{\lambda_{k}\right\}$ is ordered, i.e.; $\lambda_{0} \geqq \lambda_{1} \geqq \lambda_{2} \geqq \ldots \geqq \lambda_{k} \geqq \ldots$. From the compactness of $\mathscr{L}$ it follows that $\lambda_{k} \rightarrow 0$ as $k \rightarrow \infty$. Performing the sum over $N$, we obtain, provided that $|z|<1 /\left|\lambda_{0}\right|$, the following

$$
\begin{aligned}
\Xi(z) & =\exp \left(\sum_{\{k\}}\left\{\ln \left(1-\lambda_{k} \lambda z\right)-\ln \left(1-\lambda_{k} z\right)\right\}\right) \\
& =\prod_{\{k\}}\left(1-\lambda \lambda_{k} z\right) / \prod_{\{k\}}\left(1-\lambda_{k} z\right) \\
& =f(\lambda z) / f(z) .
\end{aligned}
$$

The infinite products in $(2.24 \mathrm{~b})$ are convergent on any compact domain of the complex plane and thus define in $(2.24 \mathrm{c})$ a ratio of two entire functions of $z$. Thus $\Xi(z)$ which is analytic in a small neighborhood of $z$, extends by analytic continuation into a meromorphic function in the entire complex plane. 
So far we have used the lattice-gas language. To translate it into the spin language we only need to redefine $\mathscr{L}$ as follows

$$
\begin{aligned}
\mathscr{L} & =\mathscr{L}_{+}+\mathscr{L}_{-} \\
\mathscr{L}_{+} f(z) & =\exp (c z) f(-\lambda+\lambda z) \\
\mathscr{L}_{-} f(z) & =\exp (-c z) f(\lambda+\lambda z) .
\end{aligned}
$$

Then all the results in this section are valid and in particular

$$
\operatorname{Tr} \mathscr{L}^{N}=1 /\left(1-\lambda^{N}\right) \sum_{\left\{\sigma_{\imath}= \pm 1\right\}} \exp \left(-c \sum_{i=0}^{N} \sigma_{i} \sum_{l=1}^{\infty} \sigma_{i+l} \lambda^{l}\right) .
$$

\section{Exponential-Polynomial Interactions}

We briefly indicate how the above analysis can be carried over to accomodate potentials of the form (1.1);

$$
\varphi(n)=\lambda^{n} \sum_{i=0}^{p} c_{i} n^{i}
$$

Ferrero [2] has shown that the operator $\mathscr{L}$ defined in (1.12) is again compact.

Proposition 3. The operator $\mathscr{L}$ defined in (1.12), acting on a Hilbert space $\mathscr{H}(D)$ of functions of $(p+1)$ variables, holomorphic on an open polydisk $D$, admits the following representation

$$
\mathscr{L}=\sum_{\left\{n_{i} ; i=0, \ldots p\right\}} \prod_{i}\left(\lambda^{n_{i}}\right) \sum_{x=0,1}\left|\varphi_{\left\{n_{i}\right\}}^{(x)}\right\rangle\left\langle\psi_{\left\{n_{i}\right\}}^{(x)} \mid \ldots\right\rangle_{\mathscr{H}}
$$

where

$$
\left|\varphi_{\left\langle n_{i}\right\}}^{(x)}\right\rangle=\exp \left(-x \tilde{c}_{0} z\right) \prod_{i=0}^{p}(A z)_{i}^{n_{1}}
$$

and

$$
\left\langle\psi_{\left\{n_{i}\right\}}^{(x)} \mid f(z)\right\rangle_{\mathscr{H}^{*}}=\oint_{\partial_{0} D} \prod_{i=0}^{p}\left(d z_{i}\right)(2 \pi i)^{-(p+1)} f\left(\left\{z_{i}\right\}\right) \mid \prod_{i=0}^{p}\left(z_{i}-\lambda x\right)^{\left(n_{i}+1\right)} .
$$

(See Section 1 for notations.) We have dropped the factor $\gamma$ in (3.2a) as this can easily be incorporated in the end if one wishes.

Proof. The representation (3.1) is an obvious generalization of Lemma 1 to $p+1$ complex variables.

$\operatorname{Tr} \mathscr{L}$ and $\operatorname{Tr} \mathscr{L}^{N}$ can be calculated in a manner analogous to the calculations in Section 2. However there are some important differences in the convergence arguments. This is best illustrated by calculating $\operatorname{Tr} \mathscr{L}$. From (3.1) and (3.2), it follows that

$$
\begin{aligned}
\operatorname{Tr} \mathscr{L}= & \sum_{\left\{n_{i}\right\}} \sum_{\{x\}} \prod_{i=0}^{p}\left(\lambda^{n_{2}}\right)\left\langle\psi_{\left\{n_{i}\right\}}^{(x)} \mid \varphi_{\left\{n_{i}\right\}}^{(x)}\right\rangle_{\mathscr{H} *} \\
= & \sum_{\left\{n_{\imath}\right\}} \sum_{(x)} \prod_{i=0}^{p}\left(\lambda^{n_{i}}\right) \oint_{\partial_{0} D^{(x)}} \prod_{\alpha}\left(d z_{\alpha}\right)(2 \pi i)^{-(p+1)} \prod_{i}\left(\sum_{j} A_{i j} z_{j}\right)^{n_{i}} \\
& \cdot \exp \left(-x \sum_{0}^{p} c_{i} z_{i}\right) / \prod_{i}\left(z_{i}-\lambda x\right)^{\left(n_{i}+1\right)} .
\end{aligned}
$$


Now the series

$$
\sum_{n_{i}=0}^{\infty}\left(\lambda \sum_{j} A_{i j} z_{j}\right)^{n_{i}} /\left(z_{i}-\lambda x\right)^{n_{i}} \quad(i=0,1, \ldots, p)
$$

are uniformly convergent provided that

$$
\left|\sum_{j} A_{i j} z_{j} /\left(z_{i}-\lambda x\right)\right|<1 / \lambda \quad(i=0,1, \ldots, p) .
$$

This is accomplished if one chooses the radii $R_{0}^{(x)}, R_{1}^{(x)}, \ldots, R_{p}^{(x)}$ of the polydisk $\partial_{0} D^{(x)}=\partial_{0} D_{0}^{(x)} \times \ldots \times \partial_{0} D_{p}^{(x)}$ such that $R_{p}^{(x)}>R_{p-1}^{(x)}>\ldots>R_{0}^{(x)}$ and

$$
R_{i}^{(x)}>\lambda /(1-\lambda)\left[x+\sum_{j=0}^{i-1}\left(\begin{array}{l}
i \\
j
\end{array}\right) R_{j}^{(x)}\right] .
$$

Thus for $x=0$ one chooses the radii $R_{i}^{(0)}$ in $(3.3 \mathrm{~b})$ such that

$$
R_{i}^{(0)}>\lambda /(1-\lambda)\left[\sum_{j=0}^{i-1}\left(\begin{array}{l}
i \\
j
\end{array}\right) R_{j}^{(0)}\right]
$$

and for $x=1$

$$
R_{i}^{(1)}>1 /(1-\lambda)\left[1+\sum_{j=0}^{i-1}\left(\begin{array}{l}
i \\
j
\end{array}\right) R_{j}^{(1)}\right] .
$$

Now the summation and integration can be interchanged and we obtain for

$$
\operatorname{Tr} \mathscr{L}=\sum_{(x)} \oint_{\partial_{0} D^{(x)}} \prod_{\alpha=0}^{p} d z_{\alpha} \exp \left(-z \sum_{i} c_{i} z_{i}\right)(2 \pi i)^{-(p+1)} / \prod_{i}\left(z_{i}-\lambda x-\lambda \sum_{j} A_{i j} z_{j}\right) .
$$

Let

$$
w_{i}=z_{i}-\lambda \sum_{j} A_{i j} z_{j} \quad(i=0,1, \ldots, p) .
$$

In matrix form

$$
W=(1-\lambda A) z \text {. }
$$

Since $(1-\lambda A)$ is non-singular, we can invert (3.9) to obtain

$$
z_{i}=\sum_{j}^{\prime} B_{i j} w_{j}
$$

where

$$
B=(1-\lambda A)^{-1} \text {. }
$$

Clearly det $B=(1-\lambda)^{p+1}$ and $B$ is again triangular matrix. The Jacobian of the transformation is easily seen to be $(1-\lambda)^{-(p+1)}$. Applying Cauchy's theorem to (3.7), the only contribution comes from the point $W=\lambda I$. Hence

$$
\begin{aligned}
\operatorname{Tr} \mathscr{L} & =(1-\lambda)^{-(p+1)} \sum_{(x)} \exp \left(-x \lambda \bar{C}(1-\lambda A)^{-1} I\right. \\
& =(1-\lambda)^{-(p+1)} \sum_{(x)} \exp \left(-\lambda x \sum_{n=0}^{\infty} \tilde{C}(\lambda A)^{n} I\right) .
\end{aligned}
$$


Now

$$
\begin{aligned}
\tilde{C} A^{n} I & =\sum_{i=0}^{p} \sum_{j=0}^{p} c_{i}\left(A^{n}\right)_{i j} \\
& =\sum_{i=0} \sum_{j=0} \sum_{k_{1}} \ldots \sum_{k_{n-1}} c_{i} A_{i k_{1}} A_{k_{1} k_{2}} \ldots A_{k_{n-1} j} \\
& =\sum_{i} \sum_{j} \sum_{\left\{k_{i}\right\}} c_{i}\left(\begin{array}{c}
i \\
k_{1}
\end{array}\right)\left(\begin{array}{c}
k_{1} \\
k_{2}
\end{array}\right) \ldots\left(\begin{array}{c}
k_{n-1} \\
j
\end{array}\right) \\
& =\sum_{i=0}^{p} c_{i}(n+1)^{i} \\
\operatorname{Tr} \mathscr{L} & =(1-\lambda)^{-(p+1)}\left\{1+\exp \left(-\sum_{n=1}^{\infty} \lambda^{n} \sum_{\alpha=0}^{p} c_{\alpha} n^{\alpha}\right)\right\} .
\end{aligned}
$$

Similarly one can calculate $\operatorname{Tr} \mathscr{L}^{N}$ and one finds the following

$$
\operatorname{Tr} \mathscr{L}^{N}=\left(1-\lambda^{N}\right)^{-(p+1)} \sum_{\left\{x_{\imath}\right\}} \exp \left(-\sum_{s=1}^{N} x_{s} \sum_{n=1}^{\infty} x_{s+n} \lambda^{n} \sum_{\alpha=0}^{p} c_{\alpha} n^{\alpha}\right) .
$$

One constructs $\Xi(z)$ analogous to (1.10)

$$
\begin{aligned}
\Xi(z) & =\exp \left(\sum_{N=1}^{\infty} z^{N} / N\left(1-\lambda^{N}\right)^{p+1} \operatorname{Tr} \mathscr{L}^{N}\right) \\
& =\exp \left(\sum_{N=1}^{\infty} z^{N} / N \sum_{\alpha=0}^{p+1}\left(\begin{array}{c}
p+1 \\
\alpha
\end{array}\right)\left(-\lambda^{N}\right)^{\alpha} \sum_{k} \lambda_{k}^{N}\right)
\end{aligned}
$$

where $\left\{\lambda_{k}\right\}$ are the eigenvalues of $\mathscr{L}$ repeated according to their multiplicity. Performing the sum over $N$, we obtain

$$
\Xi(z)=\exp \left(\sum_{\alpha=0}^{p+1}(-1)^{\alpha+1}\left(\begin{array}{c}
p+1 \\
\alpha
\end{array}\right) \ln \prod_{k}\left(1-\lambda_{k} \lambda^{\alpha} z\right)\right) .
$$

Defining $f(z)$ by

$$
f(z)=\prod_{k}\left(1-\lambda_{k} z\right)
$$

which is an entire function $z$, one obtains for 3.17

$$
\begin{aligned}
\Xi(z) & =\exp \left(\sum_{\alpha=0}^{p+1}(-1)^{\alpha+1}\left(\begin{array}{c}
p+1 \\
\alpha
\end{array}\right) \ln f\left(\lambda^{\alpha} z\right)\right) \\
& =\prod_{\alpha=0}^{p+1}\left[f\left(\lambda^{\alpha} z\right)\right]^{(-1)^{\alpha+1}\left(\begin{array}{c}
p+1 \\
\alpha
\end{array}\right) .}
\end{aligned}
$$

Thus $\Xi(z)$ is a ratio of a finite product of entire functions. Hence it is meromorphic. The approach to the transfer matrix outlined above can easily be generalized to pair potentials which are a finite sum of exponentials of the form

$$
\Phi(n)=\sum_{i=1}^{M} \lambda_{i}^{n} \sum_{j=1}^{p}\left(c_{i j} n^{j}\right)
$$


Acknowledgements. It is a pleasure to thank D. Ruelle who not only suggested the problem but also gave constant encouragement and help. My sincere thanks are also due to D. Mayer and F. Dunlop with whom I had fruitful discussions. Finally, my sincere thanks are also due to G. Gallavotti for suggesting improvements on the original version of the manuscript.

\section{References}

Ruelle, D.: Statistical mechanics of a one dimensional Lattice gas. Commun. math. Phys. 9, 267-278 (1968)

Ferrero, P.: Preprint

Schatten, R.: Norm Ideals of completely continuous operators. Berlin-Göttingen-Heidelberg: Springer 1960

Reed, M., Simon, B.: Methods of Modern Mathematical Phys. Vol. 1. London: Academic Press 1972

Communicated by G. Gallavotti

Received June 10, 1975 
